\title{
Fatores associados à independência de comunicação entre idosos da comunidade
}

Factors associated with communication independence in older adults in the community

\author{
Factores asociados a la independencia de comunicación em adultos mayores de la comunidade
}

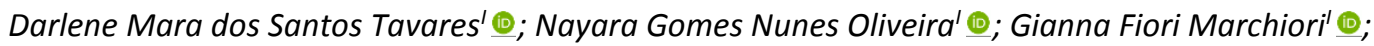 \\ Lenniara Pereira Mendes Santana' @ ; Mariana Silva Freitas Guimarães' @ ; Jussara da Cruz Jardim $@$
}

'Universidade Federal do Triângulo Mineiro, Uberaba, MG, Brasil

\begin{abstract}
RESUMO
Objetivo: descrever a média total de independência geral e por domínios de comunicação dos idosos da comunidade e verificar as variáveis sociodemográficas e de saúde associadas à maior média de independência de comunicação entre os idosos. Método: estudo transversal e analítico, desenvolvido entre 808 idosos da comunidade. Procederam-se às análises: descritiva, bivariada e regressão linear múltipla $(p<0,05)$. Resultados: verificou-se necessidade mínima de auxílio na comunicação $(6,43 \pm 0,77)$; quanto aos domínios obteve maior escore de independência de comunicação nas necessidades básicas $(6,88 \pm 0,51)$ e menor na leitura, escrita e conceitos numéricos $(5,96 \pm 1,63)$. A maior média de independência de comunicação associou-se à faixa etária $60-80(p<0,001)$; ter escolaridade $(p<0,001)$; renda maior que um salário mínimo $(p<0,001)$; à ausência do declínio cognitivo $(p<0,001)$ e do indicativo de sintomas depressivos $(p=0,014)$. Conclusão: a escolaridade, menor idade, maior renda e a ausência do declínio cognitivo e do indicativo de sintomas depressivos contribuíram para maior independência de comunicação.
\end{abstract}

Descritores: Idoso; Saúde do idoso; Enfermagem Geriátrica; Comunicação.

\section{ABSTRACT}

Objective: to describe mean independence overall and by communication domains in older adults in the community, and to ascertain the socio-demographic and health variables associated with higher mean communication independence in elderly. Method: this analytical, cross-sectional study was conducted with 808 older adults in the community. Descriptive, bivariate and multiple linear regression analyses were performed $(p<0.05)$. Results: minimum need for assistance in communication was assessed (6.43 \pm 0.77$)$; by domains, the highest communication independence score was obtained in basic needs $(6.88 \pm 0.51)$ and the lowest in reading, writing and numerical concepts (5.96 \pm 1.63$)$. Higher mean communication independence scores were associated with the $60-80$ age group $(p<0.001)$; level of schooling $(p<0.001)$; income greater than one minimum wage $(p<0.001)$; and absence of cognitive decline $(p<0.001)$ and of indications of depressive symptoms $(p=0.014)$. Conclusion: Schooling, lower age, higher income and the absence of cognitive decline and indications of depressive symptoms contributed to greater communication independence.

Descriptors: Aged; Health of the Elderly; Geriatric Nursing; Communication.

\section{RESUMEN}

Objetivo: describir la independencia media global y por dominios de comunicación en adultos mayores de la comunidad, y conocer las variables sociodemográficas y de salud asociadas con una mayor independencia media de comunicación en adultos mayores. Método: este estudio analítico y transversal se realizó con 808 adultos mayores de la comunidad. Se realizaron análisis descriptivos, bivariados y de regresión lineal múltiple $(p<0,05)$. Resultados: se evaluó la necesidad mínima de asistencia en la comunicación $(6,43 \pm 0,77)$; por dominios, la puntuación más alta de independencia comunicativa se obtuvo en necesidades básicas $(6,88 \pm 0,51)$ y la más baja en lectura, escritura y conceptos numéricos $(5,96 \pm 1,63)$. Las puntuaciones medias más altas de independencia de la comunicación se asociaron con el grupo de edad de 60 a 80 años $(p<0,001)$; nivel de escolaridad ( $p$ $<0,001)$; ingresos superiores a un salario mínimo $(p<0,001)$; y ausencia de deterioro cognitivo $(p<0,001)$ y de indicaciones de síntomas depresivos $(p=0,014)$. Conclusión: La escolaridad, la menor edad, los ingresos más altos y la ausencia de deterioro cognitivo e indicaciones de síntomas depresivos contribuyeron a una mayor independencia comunicativa.

Descriptores: Anciano; Salud del Anciano; Enfermería Geriátrica; Comunicación.

\section{INTRODUÇÃO}

O envelhecimento populacional traz novos desafios para os profissionais de saúde ${ }^{1,2}$, dentre eles a funcionalidade da comunicação ${ }^{3}$. A comunicação funcional consiste na habilidade de emitir e compreender uma mensagem de maneira independente e eficiente ${ }^{4}$. Cerca de um quinto da população brasileira com mais de 65 anos de idade apresenta incapacidade comunicativa, que pode resultar em perda de independência e sentimento de solidão 5 . 
A comunicação desempenha um papel central nas relações do indivíduo com o meio em que está inserido, constituindo-se um fator decisivo para a independência, autonomia e bem-estar dos idosos ${ }^{6}$. No âmbito da Enfermagem, a comunicação é uma estratégia essencial na atenção à saúde da população idosa, pois confere segurança e confiança do indivíduo ao profissional, para que exponha suas necessidades, fomentando, então, uma interação positiva entre o ser cuidado e o enfermeiro. ${ }^{7}$ Dessa forma, faz-se necessária a busca do conhecimento acerca da temática para direcionar o uso da comunicação nas diversas situações do cuidar ${ }^{7}$.

As condições que resultam em problemas de comunicação diferem em tipo, gravidade e na relação com outras comorbidades $^{8}$. Estudo conduzido com idosos não institucionalizados residentes nos Estados Unidos verificou que aqueles com dificuldade de comunicação eram mais velhos, com autopercepção de saúde negativa, maior número de morbidades, com maiores limitações funcionais, níveis mais altos de deficiência visual, menores redes sociais e participação em atividades sociais e maiores escores de solidão em comparação com aqueles sem dificuldade de comunicação ${ }^{9}$.

As afirmações sobre o impacto dos problemas de comunicação são complexas, pois várias condições estão associadas às limitações físicas e cognitivas, podendo afetar a autonomia e a independência dos idosos ${ }^{9}$.Assim, pesquisas com ênfase nessa temática auxiliam os profissionais de saúde no desenvolvimento de intervenções precoces, por meio do direcionamento do cuidado, como a promoção da saúde, prevenção de agravos e controle dos fatores de risco ${ }^{9}$, contribuindo com a manutenção da independência e da capacidade comunicativa, e consequentemente com a redução dos custos com os serviços de saúde ${ }^{10}$.

Nas literaturas científicas, nacional e internacional, foram identificados estudos que avaliaram o perfil da comunicação funcional de idosos saudáveis não institucionalizados ${ }^{11}$; a associação entre a comunicação social e a independência para as Atividades Instrumentais de Vida Diária entre idosos da comunidade ${ }^{3}$; com comprometimento cognitivo leve ${ }^{6}$; e em acompanhamento ambulatorial ${ }^{12}$. Outras investigações verificaram os fatores associados aos aspectos negativos das habilidades de comunicação $0^{3,8,9,13}$. Destacam-se as lacunas voltadas para a compreensão dos fatores associados aos aspectos positivos da independência de comunicação entre idosos da comunidade.

Ao considerar que a dependência de comunicação é um importante indicador da capacidade cognitiva do idoso ${ }^{14}$, que sua identificação precoce é indispensável na prevenção de incapacidades funcionais nessa faixa etária ${ }^{3}$ e que há uma escassez de estudos com essa temática, os objetivos dessa pesquisa foram descrever a média total de independência geral e por domínios de comunicação dos idosos da comunidade e verificar as variáveis sociodemográficas e de saúde associadas à maior média de independência de comunicação entre os idosos.

\section{MÉTODO}

Estudo de abordagem quantitativa, tipo inquérito domiciliar, transversal, observacional e analítico, que se desenvolveu na área urbana de um município de Minas Gerais.

Para a seleção da população da área urbana utilizou-se a amostragem por conglomerado em múltiplo estágio. O cálculo do tamanho amostral considerou um coeficiente de determinação $R^{2}=0,02$ em um modelo de regressão linear múltipla com 08 preditores, tendo como nível de significância ou erro do tipo I de $\alpha=0,05$ e erro do tipo II de $\beta=0,2$, resultando, portanto, em um poder estatístico apriorístico de 80\%. Utilizando-se o aplicativo PASS (Power Analysis and Sample Size), versão 13, introduzindo-se os valores acima descritos, obtém-se um tamanho de amostra mínimo de $\mathrm{n}=744$. Considerando uma perda de amostragem de $15 \%$ (recusas em participar), o número final de tentativas de entrevista foi de 930.

Os critérios de inclusão foram: ter 60 anos ou mais de idade, residir na zona urbana do município. Excluíram-se os idosos com declínio cognitivo avaliado pelo Miniexame do Estado Mental (MEEM) ${ }^{15}$ sem informante para responder ao Questionário de Atividades Funcionais (PFEFFER) ${ }^{16}$ e com escore final maior ou igual a seis pontos no PFEFFER; que apresentavam sequelas graves de AVE com perda localizada de força e afasia; doença de Parkinson em estágio grave ou instável com comprometimentos graves da motricidade, da fala ou da afetividade, pois impossibilitaria a realização das avaliações.

Foram entrevistados 823 idosos, dos quais 15 apresentaram declínio cognitivo. Assim, 808 idosos compuseram a amostra do estudo.

A coleta dos dados foi realizada no domicílio dos idosos, de maio (2017) a junho (2018), por meio de entrevista direta. Os dados sociodemográficos e as morbidades autorreferidas foram obtidos por meio do formulário estruturado. Para verificar a presença do indicativo de sintomas depressivos utilizou-se a Escala de Depressão Geriátrica Abreviada ${ }^{17}$. O declínio cognitivo foi mensurado por meio MEEM considerando os pontos de corte: $\leq 13$ para analfabetos, $\leq 18$ para escolaridade média (de um a 11 anos) e $\leq 26$ para alta escolaridade (superior a 11 anos) ${ }^{15}$. 
A independência de comunicação foi avaliada por meio da Escala de Avaliação Funcional das Habilidades de Comunicação (ASHA-FACS) ${ }^{18}$, aplicada ao cuidador/familiar, que é composta por quatro domínios: Comunicação Social, que está relacionado às situações sociais que exigem interação com o falante; Comunicação de Necessidades Básicas, ou seja, a reação às situações de necessidade e emergência; Leitura, Escrita e Conceitos Numéricos, que consiste na capacidade dos idosos em anotar um recado, identificar rótulos de alimentos e/ou preencher pequenos formulários; e o Planejamento Diário, que envolve a noção de agenda a cumprir e compromissos, uso do telefone e calendário. ${ }^{18} \mathrm{~A}$ ASHA-FACS é graduada como uma escala de sete pontos, que avalia o desempenho da comunicação ao longo do "continuum" de independência, em termos de níveis de assistência e/ou prontidão necessários à comunicação ${ }^{18}$. Nessa graduação, sete significa que o indivíduo tem desempenho adequado no item, sem necessidade de qualquer auxílio; seis - necessita de assistência mínima para desempenho adequado; cinco - assistência mínima a moderada; quatro assistência moderada; três - assistência de moderada a máxima; dois - assistência máxima; e um - não é capaz de determinado comportamento, mesmo com assistência máxima para isso. Ao final, calcula-se a média ponderada chegando-se ao valor médio de independência de comunicação ${ }^{18}$.

As variáveis sociodemográficas estudadas foram: sexo (feminino; masculino), faixa etária, em anos (60 l-70; 70 -80; 80 ou mais), escolaridade, em anos de estudo (nenhum; 1 -5; 5 anos ou mais), arranjo de moradia (só; acompanhado (a)) e renda mensal individual, em salários mínimos (sem rendimento; $\leq 1 ;>1$ ). Variáveis relacionadas à saúde: número de morbidades autorreferidas ( 0 -5; 5 ou mais); indicativo de sintomas depressivos (sim; não) e declínio cognitivo (sim; não) e escore de independência de comunicação.

Para realização das entrevistas, foram selecionados dez entrevistadores, os quais passaram por treinamento, capacitação e abordagem sobre questões éticas da pesquisa. Construiu-se um banco de dados eletrônico, no programa Excel $^{\circledR}$, com dupla digitação. Após a verificação das inconsistências entre as duas bases de dados, o banco de dados foi importado para o software "Statistical Package for the Social Sciences" (SPSS ) versão 22.0, para análise.

Os dados foram submetidos à análise de frequência absoluta e relativa para variáveis categóricas; e média e desvio padrão para as quantitativas. Para verificar os fatores sociodemográficos e de saúde associados à maior média de independência de comunicação foi realizada análise bivariada preliminar empregando-se o teste $t$ de Student. As variáveis de interesse que atenderam o critério estabelecido $(p \leq 0,10)$ foram introduzidas no modelo de regressão linear múltipla. Tendo como desfecho o escore de independência de comunicação e as variáveis preditoras: sexo; faixa etária; escolaridade; arranjo de moradia; número de morbidades autorreferidas; indicativo de sintomas depressivos e capacidade cognitiva. Para as análises, bivariada e regressão linear múltipla, foram dicotomizadas as variáveis: faixa etária ( 60 -80 anos; 80 anos ou mais); escolaridade (com; sem) e renda individual mensal, em salários mínimos (>1; $\leq 1)$. Considerou o intervalo de confiança de $95 \%$ e o nível de significância de $p<0,05$.

O projeto foi aprovado pelo Comitê de Ética em Pesquisa com Seres Humanos, protocolo no 2.053.520. Após a anuência do idoso e a assinatura do Termo de Consentimento Livre Esclarecido, conduziu-se a entrevista.

\section{RESULTADOS}

A maioria dos idosos era do sexo feminino (66,7\%), com 70 -80 anos de idade $(42,1 \%)$, morava acompanhada $(81,6 \%)$, com 1 -5 anos de estudo (50,4\%), renda individual mensal de até um salário mínimo $(51,0 \%)$, com cinco ou mais morbidades autorreferidas (67,5\%), sem declínio cognitivo (98,5\%) e sem indicativo de sintomas depressivos (76,5\%).

A Tabela 1 apresenta os domínios da Escala de Avaliação Funcional das Habilidades de Comunicação entre idosos comunitários.

TABELA 1: Distribuição das médias e desvio padrão dos domínios da Escala de Avaliação Funcional das Habilidades de Comunicação entre idosos de Uberaba, Minas Gerais, Brasil, 2018.

\begin{tabular}{lcc}
\hline \multicolumn{1}{c}{ Domínios } & Média & Desvio Padrão \\
\hline Comunicação social & 6,79 & 0,52 \\
Comunicação de necessidades básicas & 6,88 & 0,51 \\
Leitura, escrita e conceitos numéricos & 5,96 & 1,63 \\
Planejamento diário & 6,07 & 1,29 \\
Independência de comunicação (média total) & 6,43 & 0,77 \\
\hline
\end{tabular}

Em relação à independência de comunicação, a média total alcançada pelos idosos foi de $6,43( \pm 0,77)$ pontos, considerando-os com necessidade mínima de auxílio nessa função. Na análise de cada domínio verificou-se maior média de independência para a Comunicação das Necessidades Básicas $(6,88 \pm 0,51)$ e menor no domínio Leitura, Escrita e Conceitos numéricos $(5,96 \pm 1,63)$. 
A Tabela 2 apresenta a distribuição das variáveis sociodemográficas e de saúde, segundo escore de independência de comunicação dos idosos comunitários.

TABELA 2: Análise comparativa do escore de independência de comunicação, de acordo com as

\begin{tabular}{|c|c|c|c|}
\hline Variáveis & Média & Desvio padrão & $p^{*}$ \\
\hline \multicolumn{4}{|l|}{ Sexo } \\
\hline Masculino & 6,54 & 0,63 & 0,002 \\
\hline Feminino & 6,37 & 0,82 & \\
\hline \multicolumn{4}{|l|}{ Escolaridade } \\
\hline Com escolaridade & 6,58 & 0,66 & $<0,001$ \\
\hline Sem escolaridade & 5,68 & 0,80 & \\
\hline \multicolumn{4}{|l|}{ Faixa etária } \\
\hline $60-80$ & 6,48 & 0,75 & $<0,001$ \\
\hline 80 ou mais & 6,22 & 0,79 & \\
\hline \multicolumn{4}{|l|}{ Arranjo de moradia } \\
\hline Mora acompanhado(a) & 6,43 & 0,77 & 0,880 \\
\hline Mora só & 6,42 & 0,77 & \\
\hline \multicolumn{4}{|c|}{ Renda mensal individual (em salários mínimos) } \\
\hline$>1$ & 6,60 & 0,60 & $<0,001$ \\
\hline$\leq 1$ & 6,29 & 0,85 & \\
\hline \multicolumn{4}{|c|}{ Número de morbidades autorreferidas } \\
\hline $0-5$ & 6,48 & 0,77 & 0,192 \\
\hline 5 ou mais & 6,40 & 0,77 & \\
\hline \multicolumn{4}{|l|}{ Capacidade cognitiva } \\
\hline Sem declínio cognitivo & 6,44 & 0,75 & $<0,001$ \\
\hline Com declínio cognitivo & 5,23 & 0,86 & \\
\hline \multicolumn{4}{|c|}{ Indicativo de sintomas depressivos } \\
\hline Não & 6,48 & 0,75 & $<0,001$ \\
\hline Sim & 6,25 & 0,79 & \\
\hline
\end{tabular}

$\mathrm{Na}$ análise bivariada, as variáveis que atenderam ao critério estabelecido $(p \leq 0,10)$ e inseridas no modelo final de regressão linear múltipla foram: sexo $(p=0,002)$; escolaridade $(p<0,001)$; faixa etária $(p<0,001)$; renda individual mensal $(p<0,001)$; capacidade cognitiva $(p<0,001)$; e indicativo de sintomas depressivos $(p<0,001)$.

A Tabela 3 apresenta a associação entre a independência de comunicação e as características sociodemográficas e de saúde dos idosos comunitários.

TABELA 3: Modelo final de regressão linear múltipla da independência de comunicação entre idosos da comunidade, Uberaba, Minas Gerais, Brasil, 2028.

\begin{tabular}{lcc}
\hline Variáveis & $\boldsymbol{\beta}$ & $\boldsymbol{p}^{*}$ \\
\hline Sexo & 1 & \\
Feminino & 0,047 & 0,131 \\
Masculino & 1 & \\
Escolaridade & 0,396 & $<0,001$ \\
Sem escolaridade & & \\
Com escolaridade & 1 & \\
Faixa etária (em anos) & 0,108 & $<0,001$ \\
80 ou mais & & \\
60 -80 & 1 & \\
Renda individual mensal (em salários mínimos) & 0,107 & 0,001 \\
$\leq 1$ & & \\
$>1$ & 1 & \\
Capacidade cognitiva & 0,164 & $<0,001$ \\
Com declínio cognitivo & & \\
Sem declínio cognitivo & 1 & \\
Indicativo de sintomas depressivos & 0,076 & 0,014 \\
Sim & & \\
Não & &
\end{tabular}


A maior média de independência de comunicação foi associada à presença de escolaridade $(p<0,001)$; faixa etária 60 -80 anos $(p<0,001)$; renda mensal individual $>1$ salário mínimo $(p<0,001)$ e à ausência do declínio cognitivo $(p<0,001)$ e do indicativo de sintomas depressivos $(p=0,014)$.

\section{DISCUSSÃO}

As características sociodemográficas e de saúde dos idosos do estudo estão em consonância com estudos nacionais e internacionais desenvolvidos entre idosos da comunidade, os quais verificaram o predomínio de idosas ${ }^{3,6,9,13}$, com faixa etária de 70 -80 anos de idade ${ }^{6,9}$, vivendo com companheiro ${ }^{9}$, com 1 -5 anos de estudo ${ }^{3,13}$ e renda mensal individual de até um salário mínimo ${ }^{3,13}$, com polimorbidades ${ }^{19}$, sem declínio $\operatorname{cognitivo}{ }^{20}$ e ausência do indicativo de sintomas depressivos ${ }^{21-23}$.

Os problemas de comunicação afetam pessoas de todas as idades, entretanto, a prevalência e a complexidade dessas condições aumentam com a idade ${ }^{3}$. A média total de independência de comunicação do presente estudo foi superior à verificada nas investigações conduzidas com idosos da comunidade $(6,18 \text { pontos })^{11}$ e hospitalizados $(5,27$ pontos $)^{24}$. As limitações de comunicação associadas ao processo de envelhecimento humano afetam as atividades do cotidiano do idoso, como o acesso aos cuidados em saúde e a manutenção de papéis sociais ${ }^{8}$.Desta forma,ao se utilizar dos recursos da comunicação, o profissional de enfermagem tem condições de reconhecer precoce e amplamente as necessidades individuais do idoso e incluí-lo ao planejar, executar e avaliar a assistência a ser prestada ${ }^{10}$.

A maior média no domínio Comunicação de Necessidades Básicas diverge do estudo entre idosos da comunidade, no qual o de comunicação social foi o domínio com maior média $(6,64 \text { pontos })^{11}$. O fato de apresentarem melhor desempenho no domínio de comunicação de necessidades básicas pode expressar o papel que cumpre a linguagem oral

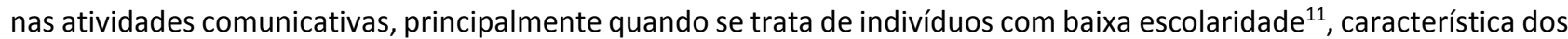
idosos do presente estudo.

Corroborando com a presente pesquisa, a menor média no domínio Leitura, Escrita e Conceitos numéricos foi verificada na investigação entre idosos da comunidade ${ }^{11}$. As habilidades de comunicação mensuradas nesse domínio consistem na capacidade dos idosos em anotar um recado, identificar rótulos de alimentos e/ou preencher pequenos formulários ${ }^{18}$, atividades que dependem de conteúdos, em geral aprendidos na escola. $A$ escolaridade média da população brasileira com 60 anos ou mais de idade é baixa, com média de 4,1 anos de estudo durante toda a vida e os dados de analfabetismo referentes a essa população são expressivos, presente em 5,1 milhões ${ }^{25}$. Diante disso, torna-se essencial que os profissionais de enfermagem considerem esse fator, que pode interferir na compreensão das informações e na adesão aos cuidados necessários para manutenção da saúde.

Concernente à associação entre a presença de escolaridade e a maior média de independência de comunicação, investigação realizada com idosos cadastrados em uma Unidade de Saúde da Família no Rio de Janeiro (RJ) verificou que $79,6 \%$ relataram apenas o ensino fundamental ${ }^{13}$.Também se identificou prevalência elevada de dificuldade em habilidade de leitura e escrita, com maior percentual $(52,8 \%)$ relacionado à dificuldade para leitura organizada ${ }^{13}$, o que pode justificar os obstáculos identificados para a independência de comunicação do idoso. Entretanto, na investigação não foi realizado análise de associação entre escolaridade e habilidades de comunicação.

Já a relação inversa, ou seja, a associação entre a presença de escolaridade e maior média de independência de comunicação pode ser explicada diante da maior capacidade dos idosos em compreender informações relacionadas à saúde, gerenciar suas necessidades básicas, elaborar um planejamento diário e integrar-se e interagir no âmbito social. É importante ressaltar que não foi identificado na literatura científica investigações sobre a associação entre escolaridade e maior média de independência de comunicação entre idosos.

No que refere ao resultado da variável faixa etária, investigação internacional com idosos de 65 anos ou mais de idade da comunidade verificou que, quando comparadas médias de idade entre os idosos com (74.15 \pm 6.23 ) e sem (77.53 \pm 6.84$)$ problemas de comunicação, os mais jovens apresentaram menores dificuldades de comunicação $(p<0,001)$; e maior rede de relações sociais $(p=0,002)^{9}$.

As mudanças nas habilidades de comunicação são resultado do processo normal de envelhecimento humano, podendo ocorrer concomitante à presença de doenças crônicas. Por isso, essas perdas e alterações são comumente identificados em idosos com idade mais avançada ${ }^{8}$, enquanto aqueles na faixa etária mais jovem apresentam maiores médias de independência na comunicação e corrobora com os resultados encontrados na presente pesquisa.

A independência de comunicação também pode ser um importante indicador da capacidade cognitiva do idoso, refletindo aspectos preservados ou alterados ${ }^{14}$. Essa relação entre as dificuldades de comunicação e o declínio cognitivo 
na população idosa já foi mencionada na literatura científica ${ }^{6,14}$, entretanto, não foram identificados estudos que verificaram a associação entre a independência de comunicação e o declínio cognitivo nesse grupo populacional.

O declínio cognitivo pode causar comprometimento significativo da memória, aprendizagem, linguagem, orientação, funções executivas, velocidade de processamento de informações, autonomia e independência funcional e de comunicação, gerando prejuízos na qualidade de vida e na saúde dessa população ${ }^{26,27}$. Idosos com declínio cognitivo apresentam alterações que interferem na comunicação, tais como, dificuldades em se referir aos familiares pelo nome, solicitar informações sobre pessoas ou acontecimentos e a compreensão de conversas em ambientes barulhentos e do que assiste na televisão ou ouve no rádio ${ }^{6}$.

Estudo desenvolvido entre idosos inscritos no programa da Universidade Aberta à Terceira Idade, programa da Universidade Federal de Pernambuco evidenciou relação significativa entre a independência para as atividades instrumentais da vida diária e os quatro domínios da comunicação social, a saber: nomeia pessoas familiares pelo nome $(p=0,003)$; solicita informação sobre pessoas ou acontecimentos $(p=0,035)$; compreende conversas em ambiente barulhento $(p=0,0448)$ e o que assiste na televisão ou ouve no rádio $(p=0,012)^{6}$.

Assim, a incapacidade comunicativa é considerada importante causa de perda ou restrição da participação social, comprometendo a capacidade de execução das decisões tomadas, afetando diretamente a independência do indivíduo $0^{5,6,28}$

O isolamento da pessoa idosa, muitas vezes relacionado ao declínio na qualidade de sua comunicação, pode gerar um impacto psicossocial no idoso. Por isso, vale ressaltar que a saúde da comunicação constitui um importante determinante para o envelhecimento ativo, uma vez que permite a inclusão e interação social do idoso, evitando o isolamento e o surgimento de sintomas depressivos ${ }^{3,13}$. Destaca-se que não foram encontrados nas literaturas, nacional e internacional, estudos que verificaram a associação entre a independência de comunicação e a presença do indicativo de sintomas depressivos.

Além disso, a comunicação não engloba apenas aspectos como troca de informações, mas também é considerada uma importante ferramenta de estabilização e manutenção da vida social, além de importante fator para obtenção de cuidados em saúde ${ }^{8}$.Neste contexto, a avaliação das habilidades de comunicação torna-se imprescindível para que o idoso consiga manter ou melhorar funções relacionadas à socialização, bem estar e manutenção da autonomia funcional, e com isso otimizar sua qualidade de vida ${ }^{13}$.

Visto que a independência de comunicação é uma ferramenta essencial no cuidado em saúde, torna-se necessária a sua avaliação durante a consulta de enfermagem gerontológica, para o direcionamento do plano de cuidados. Ademais, os enfermeiros da atenção primária são os profissionais que possuem maior contato com os idosos da comunidade, assim, a identificação dos fatores sociodemográficos e de saúde associados à independência de comunicação dessa população auxilia no planejamento e implementação de ações de enfermagem direcionadas a essas condições, em busca do bem-estar e melhoria na qualidade de vida.

Em contrapartida, há limitações quanto ao delineamento transversal, que não permite estabelecer relação de causalidade entre os fatores investigados e à exclusão de idosos com comprometimento cognitivo que pode ter favorecido uma amostra mais saudável, no entanto, a possibilidade de viés de seleção foi minimizada, uma vez que todos os idosos elegíveis foram entrevistados.

\section{CONCLUSÃO}

Os idosos apresentaram necessidade mínima de auxílio para comunicação, com maior independência no domínio das necessidades básicas e pior na leitura, escrita e conceitos numéricos. A presença de escolaridade, assim como, a menor idade, maior renda e a ausência do declínio cognitivo e do indicativo de sintomas depressivos associaram-se à maior média de independência de comunicação.

Os resultados revelam a necessidade de uma compreensão ampliada dos fatores associados à independência de comunicação de idosos da comunidade, em especial aqueles com idade avançada e baixa escolaridade e renda, pois a sua ocorrência é influenciada por aspectos multifatoriais que variam conforme o contexto em que os indivíduos estão inseridos. Ademais, o declínio cognitivo e o indicativo de sintomas depressivos são aspectos que devem ser considerados na elaboração de estratégias de enfermagem direcionadas a este segmento populacional, já que são fatores passíveis de intervenções desses profissionais de saúde.

\section{REFERÊNCIAS}

1. World Health Organization. Global health observatory data repository. Retrieved. 2015. [cited 2020 May 25] Available from: http://apps.who.int/gho/data/view.main.700?lang=en 
2. Barris MJ, Fernandes AA. Active ageing promotion at local level: Analyzing municipal programs. Rev. Port. Saúde Pública [Internet]. 2014; [cited 2020 May 25]; 32(2):188-96. DOI: https://doi.org/10.1016/j.rpsp.2014.09.002

3. Coutinho ATQ, Vilela MBR, Lima MLLT, Silva VL. Social communication and functional independence of the elderly in a community assisted by the family health strategy. Rev. CEFAC [Internet]. 2018 [cited 2020 May 25]; 20(3):363-73. DOI: http://dx.doi.org/10.1590/1982-0216201820313417

4. American Speech-Language-Hearing Association (ASHA). Advisory report, functional communication measures project. Rockville (MD). 1990 [cited 2020 may 25]; DOI: https://doi.org/10.1007/978-3-319-56782-2_854-4

5. Moraes EM, Pereira AMVB, Azevedo RS, Moraes FL. Avaliação multidimensional do idoso. Secretaria de estado da saúde do Paraná. Curitiba: SESA [Internet]. 2018 [cited 2020 May 25]; 113. Available from: https://www.saude.pr.gov.br/sites/default/arquivos_restritos/files/documento/202004/avaliacaomultiddoidoso_2018_atualiz.pdf

6. Sobral AIGP, Araujo CMT, Sobral MFF. Mild cognitive impairment in the elderly Relationship between communication and functional capacity. Dement. Neuropsychol. [Internet]. 2018 [cited 2020 May 25]; 12(2):165-72. DOI: http://dx.doi.org/10.1590/1980-57642018dn12-020009

7. Silva JPG, Costa KNFM, Silva GRF, Oliveira SHS, Almeida PC, Fernandes MG. Nursing consultation for the elderly: instruments of communication and nursing roles according to Peplau. Esc. Anna Nery Rev. Enferm. [Internet]. 2015 [cited 2020 May 25]; 19(1):154-61. DOI: https://www.scielo.br/scielo.php?pid=S1414-81452015000100154\&script=sci_arttext\&tlng=en

8. Yorkston KM, Bourgeois MS, Baylor CR. Communication and Aging. Phys. Med. Rehabil. Clin. N. Am [Internet]. 2010 [cited 2020 May 25]; 21(2):309-19. DOI: http://dx.doi.org/ 10.1016/j.pmr.2009.12.011

9. Palmer AD, Newsom JT, Rook KS. How does difficulty communicating affect the social relationships of older adults? An exploration using data from a national survey. Journal of communication disorders [Internet]. 2016 [cited 2020 May 25]; 62:131-46. DOI: http://dx.doi.org/ 10.1016/j.jcomdis.2016.06.002

10. Schimidt TCG, Duarte YAO, Silva MJP. Mediate evaluation of replicating a Training Program in Nonverbal Communication in Gerontology. Rev. enferm. USP [internet]. 2015 [cited 2020 May 25]; 49(2):309-16. DOI: https://doi.org/10.1590/S0080623420150000200017

11. Garcia FHA, Mansur LL. Functional communication assessment: the healthy elderly. Acta Fisiatria [Internet]. 2006 [cited 2020 May 25]; 13 (2):87-9. Available from: http://www.revistas.usp.br/actafisiatrica/article/view/102591

12. Santos, DT. Funcionalidade global, da deglutição e da comunicação de idosos com comprometimento cognitivo avançado [Master thesis]. Programa de Ciências da Reabilitação da Faculdade de Medicina, Universidade de São Paulo. 2018. [cited 2020 May 25]; DOI: https://doi.org/10.11606/D.5.2019.tde-07022019-092504

13. Santiago LM, Graça CML, Rodrigues COM et al. Characterization of the elderly health in a Speech and Language Therapy perspective. Rev. CEFAC [Internet]. 2016 [cited 2020 May 25]; 18(5):1088-96. DOI: http://dx.doi.org/10.1590/1982021620161855016

14. Johnson MA, Lin F. Communication difficulty and relevant interventions in mild cognitive impairment: implications for neuroplasticity. Top Geriatr. Rehabil. [Internet]. 2014 [cited 2020 May 25]; 30(1) 18-34. DOI: http://dx.doi.org/10.1097/TGR.0000000000000001

15. Bertolluci, PHF; Brucki, SMD; Campacci, SR. et al. The Mini-Mental State Examination in an outpatient population: influence of literacy. Arquivos de Neuro-psiquiatria São Paulo [Internet]. 1994 [cited 2020 May 25]; 52(1):1-7. DOI: http://dx.doi.org/10.1590/S0004-282X1994000100001

16. Ministério da Saúde (Br). Secretaria de Atenção à Saúde. Departamento de Atenção Básica. Envelhecimento e saúde da pessoa idosa. Brasília-DF. [Internet]. 2007: 192. [cited 2020 May 25]. Available from: http://bvsms.saude.gov.br/bvs/publicacoes/abcad19.pdf

17. Almeida, OP; Almeida, SA. Reliability of the Brazilian version of the Geriatric Depression Scale (GDS) short form. Arquivos de Neuropsiquiatria São Paulo [Internet]. 1999 [cited 2020 May 25]; 57(2-B):421-6. DOI: http://dx.doi.org/10.1590/S0004282X1999000300013

18. Carvalho IAM, Mansur LL. Validation of ASHA FACS-Functional Assessment of Communication Skills for Alzheimer Disease Population. Alzheimer Dis. Assoc. Disord. [Internet]. 2008 [cited 2020 May 25]; 22(4):375-81. DOI: https://www.ncbi.nlm.nih.gov/pubmed/19068501

19. Oliveira YCA, Celino SDM, Costa GMC. Communication as an essential tool for deaf people's health care. Physis Revista de Saúde Coletiva [Internet]. 2015 [cited 2020 May 25]; 25(1):307-20. DOI: http://dx.doi.org/10.1590/S0103-73312015000100017

20. Cruz DT, Cruz FM, Ribeiro AL, Veiga CL, Leite ICG. Association between cognitive ability and the occurrence of falls in elderly. Cad. saúde coletiva [Internet]. 2015 [cited 2020 May 25]; 23(4):386-93. DOI: http://dx.doi.org/10.1590/1414$462 \times 201500040139$

21. Sengupta $P$, Benjamin Al. Prevalence of depression and associated risk factors among the elderly in urban and rural field practice areas of a tertiary care institution in Ludhiana. Indian. J. Public Health [Internet]. 2015 [cited 2020 May 25]; 59(1):3-8. DOI: https://doi.org/10.4103/0019-557X.152845

22. Aragão EIS, Campos MR, Portugal FB, Gonçalves DA, Mari JJ, Fortes SLCL. Social Support patterns in Primary Health Care: differences between having physical diseases or mental disorders. Ciênc. saúde coletiva [Internet]. 2018 [cited 2020 May 25] 23(7):2339-50. DOI: http://dx.doi.org/10.1590/1413-81232018237.21012016

23. Mendes-Chiloff CL, Lima MCP, Torres AR, Santos JLF, Duarte YO, Lebrão ML et al. Depressive symptoms among the elderly in São Paulo city, Brazil: prevalence and associated factors (SABE Study). Rev. Bras. Epidemiol [Internet]. 2018 [cited 2020 May 25]; 21(2):e180014. DOI: http://dx.doi.org/10.1590/1980-549720180014.supl.2 
24. Pereira EEB, Souza ABF, Carneiro SR, Sarges ESNF. Global functionality of hospitalized elderly. Rev. Bras. Geriatr. Geront [Internet]. 2014 [cited 2020 May 25]; 17(1):165-76.Available from: http://www.redalyc.org/articulo.oa?id=403838834016

25. Instituto Brasileiro de Geografia e Estatística (IBGE). Mudança demográfica no Brasil no início do século XXI. Subsídios para as projeções das populações, 2016. [cited 2020 May 25]. Available from: https://biblioteca.ibge.gov.br/visualizacao/livros/liv93322.pdf

26. Stoyanova II. Ghrelin: a link between ageing, metabolism and neurodegenerative disorders. Neurobiol. Dis. [Internet]. 2014 [cited 2020 May 25]; 20(72):72-83. DOI: http://dx.doi.org/10.1016/j.nbd.2014.08.026

27. Xavier AJ, D'orsi E, Oliveira CM, Orrell M, Demakakos P, Biddulph JP et al. English Longitudinal Study of Aging: Can Internet/Email Use Reduce Cognitive Decline? J. Gerontol. A. Biol. Sci. Med. Sci [Internet]. 2014 [cited 2020 May 25]; 69(9): 1117-21. DOI: http://dx.doi.org/ 10.1093/gerona/glu105

28. Keller-Cohen D, Fiori K, Toler A, Bybee D. Social relations, language and cognition in the "oldest old". Age Society [Internet]. 2006 [cited 2020 May 25]; 26(4):585-605. DOI: https://doi.org/10.1017/S0144686X06004910 